\title{
A weak acceleration effect due to residual gravity in a multiply connected universe
}

\author{
B. F. Roukema ${ }^{1}$, S. Bajtlik ${ }^{2}$, M. Biesiada ${ }^{3}$, A. Szaniewska ${ }^{1}$, and H. Jurkiewicz ${ }^{1}$ \\ 1 Toruń Centre for Astronomy, N. Copernicus University, ul. Gagarina 11, 87-100 Toruń, Poland \\ e-mail: boud@astro.uni.torun.pl \\ 2 Copernicus Astronomy Centre, ul. Bartycka 18, 00-716 Warsaw, Poland \\ 3 Astronomy \& Cosmology Department, University of Silesia, Uniwersytecka 4, 40-007 Katowice, Poland
}

Received 7 February 2006 / Accepted 29 November 2006

\begin{abstract}
Context. Understanding dark energy and measuring the topology of the Universe are two of the biggest open questions in physical cosmology. It was previously shown that multiple connectedness, via the twin paradox of special relativity, provides a novel physical justification for an assumption of the standard FLRW model: it implies a favoured space-time splitting (comoving coordinates). Aims. Could cosmic topology also imply dark energy?

Methods. We use a weak field (Newtonian) approximation of gravity and consider the gravitational effect from distant, multiple copies of a large, collapsed (virialised) object today (i.e. a massive galaxy cluster), taking into account the finite propagation speed of gravity, in a flat, multiply connected universe, and assume that due to a prior epoch of fast expansion (e.g. inflation), the gravitational effect of the distant copies is felt locally, from beyond the naïvely calculated horizon.

Results. We find that for a universe with a $\mathbb{T}^{1} \times \mathbb{R}^{2}$ spatial section, the residual Newtonian gravitational force (to first order) provides an anisotropic effect that repels test particles from the cluster in the compact direction, in a way algebraically similar to that of dark energy. For a typical test object at comoving distance $\chi$ from the nearest dense nodes of the cosmic web of density perturbations, the pressure-to-density ratio $w$ of the equation of state in an FLRW universe, is $w \sim-(\chi / L)^{3}$, where $L$ is the size of the fundamental domain, i.e. of the Universe. Clearly, $|w| \ll 1$. For a $\mathbb{T}^{3}$ spatial section of exactly equal fundamental lengths, the effect cancels to zero. For a $\mathbb{T}^{3}$ spatial section of unequal fundamental lengths, the acceleration effect is anisotropic in the sense that it will tend to equalise the three fundamental lengths.

Conclusions. Provided that at least a modest amount of inflation occurred in the early Universe, and given some other conditions, multiple connectedness does generate an effect similar to that of dark energy, but the amplitude of the effect at the present epoch is too small to explain the observed dark energy density and its anisotropy makes it an unrealistic candidate for the observed dark energy.
\end{abstract}

Key words. gravitation - cosmology: theory - cosmology: cosmological parameters

\section{Introduction}

Two of the biggest open questions in physical cosmology are (1) the interpretation of the cosmological constant (or some other form of dark energy), and (2) the global shape of the Universe, including both curvature and topology. The former is empirically known to exist from many different, more or less independent, observational approaches (the cosmic concordance model, with matter density $\Omega_{\mathrm{m}} \approx 0.3$, cosmological constant/dark energy $\Omega_{\Lambda} \approx 0.7$, Hubble constant $H_{0} \approx 70 \mathrm{~km} \mathrm{~s}^{-1} \mathrm{Mpc}^{-1}$, baryon density $\Omega_{\mathrm{b}} \approx 0.05$, (e.g. Lahav \& Liddle 2004), and observations have also suggested an answer to the latter, but the evidence is far from being conclusive, and, in principle, a final answer may be totally beyond the reach of any observations.

The analysis of empirical data that suggests the shape of the Universe consists of recent analyses of the cosmic microwave background observations by the WMAP satellite. These analyses found the temperature fluctuation map to be better modelled by a multiply-connected model of the Universe, for a Poincaré dodecahedral space (PDS) as the 3-manifold of comoving space, rather than by an "infinite" flat space (e.g. Luminet et al. 2003; Roukema et al. 2004; Aurich et al. 2005a,b; Gundermann 2005).

These analyses may or may not be supported by future observations. One of the most independent tests of the
PDS hypotheses will be high precision estimates of the total density parameter $\Omega_{\text {tot }}$. The hypotheses of these authors geometrically require $\Omega_{\text {tot }}$ to be strictly greater than unity and $\Omega_{\text {tot }}$ should lie in the predicted range $1.01 \lesssim \Omega_{\text {tot }} \lesssim 1.02$ (different authors using different methods make slightly different predictions). Future observations which yield, for example, $\Omega_{\text {tot }}=$ $1.001 \pm 0.001$, would rule out these hypotheses to a significance level of $9 \sigma$.

Independently of the present hypotheses, the question of the physical consequences of cosmic topology will remain.

We know that locally, geometry and density are directly related, via general relativity. But we have very few hints as to how global geometry should relate to other physical properties. General relativity is a local theory, not a global one.

One known effect is that cosmic topology defines the comoving reference frame.

It was shown by Uzan et al. (2002) and Barrow \& Levin (2001) that resolving the twin paradox of special relativity in a multiply-connected universe is different than in simply connected Minkowski space. Moreover, multiple connectedness implies a favoured space-time splitting. The authors show that this must correspond to the comoving reference frame.

In other words, this provides a global geometrical motivation for Weyl's postulate, i.e. the postulate that "the world lines of 
the galaxies form a three-bundle of non-intersecting geodesics orthonormal to a series of space-like hypersurfaces".

Could it be possible that cosmic topology could also provide a simple explanation for the observed density of dark energy?

If there is such a mechanism, then this would most naturally be able to explain the fact why dark energy starts to dominate at the present epoch if this mechanism were linked to a property of the present cosmological epoch.

One property of the present epoch is that density perturbations have collapsed due to gravity and formed virialised structures, of which the most dense at the present epoch are galaxy clusters, the largest which typically are of mass $M \sim 10^{15} M_{\odot}$, which are formed from most of the material within a region of linear comoving size $\sim 10-20 \mathrm{Mpc}$.

What property of virialised structures is different between the simply connected case and the multiply connected case?

The difference is that in the covering space (apparent space), the spatial distribution of distant objects is homogeneous and uncorrelated with the local distribution if space is simply connected, while if space is multiply connected, then the spatial distribution of distant objects in the covering space is not random.

Given the non-random distribution of multiple topological images of objects such as clusters in the covering space, and the possibility that there was causal contact between them at some time in the past (e.g. due to some moderate amount of inflation), is it possible that the residual gravitational effect due to distant, multiple topological images of a single object yields a gravitational effect different from that normally expected from the assumption of large-scale unperturbed, homogeneity?

In Sect. 2, the assumptions made in considering this gravitational effect, in the covering space, between multiple copies of a "small", massive object, perturbed from its initial position, are listed.

In Sect. 3, the effect is calculated.

In Sect. 4, the results of this calculation are discussed, and in Sect. 5 we conclude.

For a short, concise review of the terminology, geometry and relativistic context of cosmic topology, see Roukema (2000) (this is now slightly outdated, but is sufficient for beginners). For in-depth review papers see, e.g. Lachièze-Rey \& Luminet (1995); Luminet (1998); Starkman (1998); Luminet \& Roukema (1999); workshop proceedings are in Starkman (1998) and following articles, and Blanlœil \& Roukema (2000). For comparison and classification of different observational strategies, see e.g. Uzan et al. (1999); Luminet \& Roukema (1999); Roukema (2002); Rebouças \& Gomero (2004). What might be considered as one of the most striking theoretical results, in the sense of providing a direct link between the FLRW model and multipleconnectedness, is the implication of a favoured reference frame, which must coincide with the comoving reference frame: see Barrow \& Levin (2001) and Uzan et al. (2002).

Note that some previous work has been done on possible links between topology and dark energy, via the Casimir effect. See e.g. Ahmadi \& Nouri-Zonoz (2005) and references therein. Lachièze-Rey (1999) estimated that for $\mathrm{a} \mathbb{T}^{1}$ topology, the effect would be at least 50 orders of magnitude too weak.

\section{Assumptions and calculational choices}

Initially, in Sect. 2.1, we consider the self-gravity of a cluster to itself, provided that the cluster has been perturbed from its initial, comoving position. In Sect. 2.2, the more realistic case of essentially stationary (in comoving coordinates) clusters distributed in the nodes of the cosmic web and the effect that their multiple images have on small "test objects" nearby, is considered.

\subsection{Self-gravity of a large cluster}

The following assumptions and choices are made:

(1) Newtonian approximation of gravity;

(2) a flat covering space, $R^{3}$;

(3) calculations are made in the covering space;

(4) a region at least a few times the size of the injectivity diameter [assumed to be on a size scale approximately similar to that of the diameter of the surface of last scattering (SLS)] has been in causal contact (e.g. due to inflation);

(5) the gravitational potential induced from density perturbations at large, supra-SLS distance scales mostly cancels out due to homogeneity, but since multiple topological images are non-randomly distributed, their total contributions to the potential may not fully cancel and must be calculated explicitly;

(6) up to a few tens of Mpc around a big cluster, the only nonuniform, long-distance contributions to the local potential are from its multiple topological images;

(7) time lapse assumption: the potential from these distant images corresponds to the state of these images as they were at cosmological time $t_{1}$, about a Hubble time in the past $\left(t_{1}<t_{\text {recombination }} \ll t_{0}\right)$; at that cosmological time (possibly pre-inflation, see (4) above), these images consisted of not-yet-collapsed density perturbations, which had not yet had time to be significantly displaced towards other dense regions - both this assumption and the following (8) make the physically standard assumption that the speed of transmission of gravitational waves $c_{\mathrm{GW}}$ is equal to that of the special and general relativistic space-time constants and the electromagnetic transmission speed: $c_{\mathrm{GW}}=c_{\mathrm{ST}}=c_{\mathrm{GR}}=c_{\mathrm{EM}}$; see Ellis \& Uzan (2005);

(8) time lapse corollary: if the cluster is spatially offset (perturbed) from its original position, then the long-range contributions to the gravitational potential are only felt locally after at least a Hubble time: on a time scale much less than a Hubble time, local calculations can validly assume that only the cluster moves, not its topological images, since they have not yet received the information that the topological images have moved (see Fig. 1).

(1), (2): Assumptions (1), (2) are identical to those made for most cosmological $N$-body simulations of galaxy formation in the FLRW model, e.g. Roukema et al. (1997), Bagla (2005) and references therein. In other words, the "volume effect" and the "backreaction effect" are considered negligible - see Buchert \& Carfora (2003) for the basic equations. (The volume and backreaction effects correct for the fact that the Universe does not have an exactly homogeneous FLRW metric, it is only approximately FLRW.)

(3): Particle-Mesh (PM) cosmological $N$-body codes of galaxy formation (and also those which combine PM on a large scale with alternatives on smaller scales) almost always assume a spatial 3-manifold which is the three-torus, $\mathbb{T}^{3}$, so calculations are made in the fundamental domain - there is no need to use the covering space, so point (3) is not needed for these type of $N$-body codes.

Most direct $N$-body codes and tree codes (TC) also assume a 3-torus model, but make calculations in the covering space rather than in the fundamental domain. This is the choice (3) 


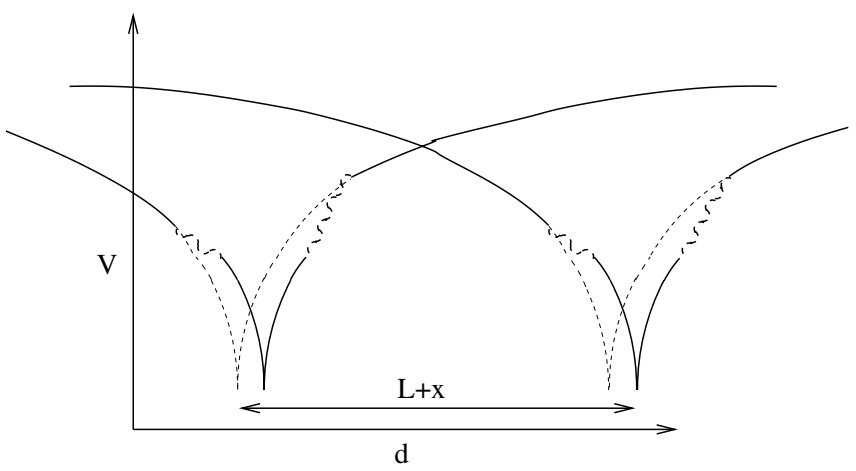

Fig. 1. Schematic diagram showing the potential to a single, massive object in a multiply connected universe of comoving size $L$, which is perturbed to the right (of the figure) by a small physical distance $x$. The information about the changes in the potential generated by this object travels outward from the object at a finite speed, $c_{\mathrm{GW}}$. If $L$ is large (about a Hubble length in size), then at or close to the position of the object, the potential due to the (distant) topological image remains that of the unperturbed topological image, until a Hubble time has passed. Anthropomorphically, we could say that during about a Hubble time following the perturbing event, the object "does not believe" that its topological image has been perturbed. Solid thick curves show the perturbed potentials, dashed thick curves show the original potentials, wavy thick curves symbolically show the information about the changed position of the object being transmitted by gravitational waves.

made here: calculations in the covering space are (usually) geometrically simpler in the covering space than in the fundamental domain.

(4): If there is no causal contact beyond the SLS, then no effect from the topologically lensed images can occur. Here, the case that the causal radius is much greater than the radius of the SLS, e.g. due to an earlier, moderate amount of inflation, is considered. Linde (2004) has recently argued that for zero or negative curvature, multiply-connected universes are more likely than simply-connected universes, and that these multiply-connected universes are expected to have undergone a moderate amount of inflation.

(5), (6): While the assumption that most large-distance effects on the potential should approximately cancel each other is likely to be a good approximation, the possible non-cancelling of the potential due to large-distance multiple images of a single cluster is likely to be of a similar order of magnitude to the effects which we are assuming to cancel under assumptions (5) and (6).

Nevertheless, we are interested in investigating whether any long-distance gravitational effect, in addition to contributions from local inhomogeneities, occurs due to topological imaging.

If the result were a large effect, then we would have to verify that it is fully self-consistent with the effects from "randomly" distributed objects.

To some degree, we could expect that the effect has already been partially modelled in PM and TC cosmological $N$-body simulations, without making the assumptions (5) and (6). However, these simulations generally make "realistic" assumptions for long-distance gravitational effects, which mean some combination of assuming long-distance homogeneity and assuming an infinite speed $c_{\mathrm{GW}}$ of the transmission of gravity, i.e. ignoring assumptions (7) and (8).

(7): The time lapse between transmission of information from a gravitational source (density perturbation) and its arrival at a "target" point in comoving space is normally ignored in $N$-body simulations of galaxy formation: gravity is assumed to be transmitted instantaneously. This is usually a reasonable approximation, since the gravitational effect from distance sources can generally be approximated (e.g. as in the top-down tree code simulations) by considering the mass in a large, distant cube of space, which subtends a small angle at the "target" point, as a single, very massive point object.

However, for topological gravity effects, we do take into account this time lapse.

(8): The initial density perturbations from which a cluster formed are "not aware" of the fact that the cluster later on collapses gravitationally and moves towards a neighbouring potential well.

Assumption (8) gives us the first of the two effects which we will calculate.

First consider a static cluster, which does not move. This is separated from one of its "adjacent" topological images by a "generator" $g_{x}$. In general, this is an isomorphism in the covering space. In the simplest 3 -torus case, it is a translation, and can be thought of as a vector in Euclidean 3-space (the covering space). It is also separated from one of its adjacent topological images in the opposite direction, i.e. by the generator $-g_{x}$.

Now consider a cluster which has moved a small distance from its "initial" location in comoving space a short time ago. The contributions to the gravitational potential, near to this cluster, from the other topological images - which are distant - remain the same as they were before the perturbation occurred. This is shown in Fig. 1.

If we consider the potentials close to the object to be the potentials relevant for making local calculations about acceleration (or worldlines), then similarly to the way luminosity distance $d_{L}$ is defined in terms of the observational flux $f$ and intrinsic luminosity $L, f=L /\left(4 \pi d_{L}^{2}\right)$, we can define the effective comoving distance $d_{\mathrm{eff}}$ (for a closer analogy with luminosity distance, we could also call this the "gravity distance") in terms of the intrinsic mass $m$ and the component of locally felt acceleration $\ddot{x}$ due to the distant object (the "observed" gravitational acceleration), i.e. $d_{\mathrm{eff}}$ is the distance satisfying

$\ddot{x}=-\frac{G m}{d_{\mathrm{eff}}^{2}}$.

In other words, $d_{\mathrm{eff}}$ is the comoving distance to a distant object implied by the local shape of its potential, taking into account the standard value of $c_{\mathrm{GW}} \approx 3 \times 10^{8} \mathrm{~m} \mathrm{~s}^{-1}$ rather than the naïve Newtonian approximation $c_{\mathrm{GW}}=\infty$.

Using this concept of distance, a cluster which has moved from its "initial" location in comoving space is nearer to one (or two or three, depending on the direction of motion) of its topological images, since in standard physics, $c_{\mathrm{GW}}$ is finite.

Hereafter, we use the effective comoving distance unless otherwise stated.

Let us consider the component of its motion towards one of its three adjacent images, so that the cluster is displaced by distance $x$ (in "physical" coordinate units) from its "initial" location in the direction of generator $g_{x}$ of comoving length $L$, as shown in Fig. 2. Since we assume Newtonian gravity at the present epoch, $L$ is also the relevant distance in proper units.

Given points (1) to (8), we now have a cluster which feels unequal gravitational pulls from a pair of its closest topological images: it is slightly less than L from one image, and slightly more than L from the opposite image. The net result should be a gravitational pull towards the former.

This self-gravity effect should be absent in any $N$-body simulation which assumes gravity is transmitted instantaneously, 


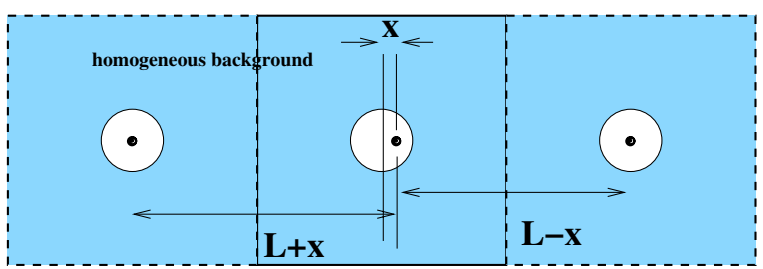

Fig. 2. Self-gravity of a large cluster. A flat, toroidal Universe model of comoving side length $L$, filled with what is assumed to be a homogeneous density field except for one massive, collapsed object (e.g. cluster of galaxies) shown as a black spot surrounded by an empty sphere from which the matter forming it was originally distributed. This object is slightly perturbed, by physical distance $x$, from its original position towards one of its adjacent virtual copies in the comoving ("apparent") space. Since the potential in the "central" copy of the fundamental domain is determined by the two adjacent copies of the fundamental domain, the object "perceives" the adjacent topological images in their original positions.

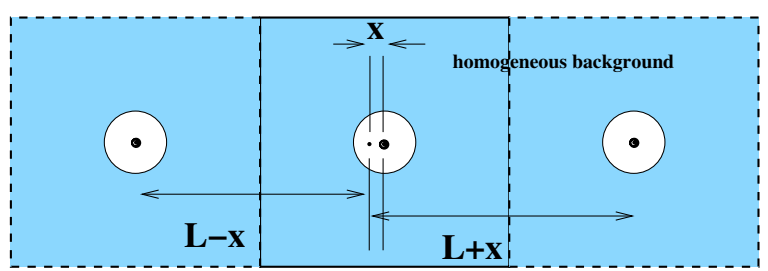

Fig. 3. Effect relative to the cosmic web. As in Fig. 2, except that the big black spot representing a massive, collapsed object is no longer perturbed, and instead, a small test object located physical distance $x$ from the cluster, along the line separating the two clusters, is shown.

since the effects of the two adjacent topological images will always perfectly cancel if $c_{\mathrm{GW}}=\infty$ is assumed.

\subsection{Effect relative to the cosmic web}

We know from linear perturbation theory of the collapse of linear overdensities in an FLRW universe, such as the Zel'dovich approximation and $\mathrm{N}$-body simulations, that matter generally "falls" from low density regions (voids) into filaments and streams along filaments towards knots where the filaments join together into what correspond today to massive galaxy clusters.

In general, the less massive objects move faster than the higher mass objects, due to conservation of momentum (Newtonian): relatively low mass objects fall (in comoving coordinates) towards the massive galaxy clusters at the "knots" of the cosmic web, while the most massive clusters have relatively little peculiar velocities with respect to the comoving frame.

In this case, we can consider a massive cluster which is approximately stationary and $x$ to be the distance remaining between a test particle (of negligible mass) and the cluster. The test particle is so far mostly comoving with the comoving reference frame, i.e. its peculiar velocity (velocity relative to the comoving frame), at which it falls towards the cluster is "small".

The same assumptions and choices, (1) to (7), are made as in Sect. 2.1. Since the cluster is considered stationary, (8) is no longer relevant.

The geometry in this case is that shown in Fig. 3.

\section{Calculation and results}

\subsection{One-dimensional analysis}

For simplicity, we first make a one-dimensional analysis, i.e. we consider multiple images only in one direction, effectively assuming a $\mathbb{T}^{1} \times \mathbb{R}^{2}$ spatial hypersurface, hereafter, written $\mathbb{T}^{1}$.

\subsubsection{Self-gravity of a large cluster}

Figure 2 shows the geometry of the situation based on the assumptions and general calculation choices of Sect. 2, for a 1 -torus, $\mathbb{T}^{1}$ model, where, for simplicity, we assume that the cluster is moving directly towards one of its adjacent images, rather than at an arbitrary direction. A more accurate calculation would only modify the present calculation by less than an order of magnitude.

The Newtonian attractive force towards the slightly closer of the two topological images of the adjacent cluster, i.e. towards the right in Fig. 2, is then:

$F \approx G m^{2}\left[\frac{1}{(L-x)^{2}}-\frac{1}{(L+x)^{2}}\right]$

where $m$ is the mass of the cluster, $G$ is the Newtonian gravitational constant, $L$ is the comoving size of the fundamental domain in the chosen direction, and $x \ll L$ is the displacement in the direction of the closest topological image, in physical coordinate units. By the time lapse assumption (7), the comoving distance $L$ is

Let us define

$\epsilon:=\frac{x}{L}$

Then

$$
\begin{aligned}
F & \approx G \frac{m^{2}}{L^{2}}\left[\frac{1}{(1-\epsilon)^{2}}-\frac{1}{(1+\epsilon)^{2}}\right] \\
& \approx G \frac{m^{2}}{L^{2}}\left[\left(1+2 \epsilon+3 \epsilon^{2}+\ldots\right)-\left(1-2 \epsilon+3 \epsilon^{2}+\ldots\right)\right] \\
& =G \frac{m^{2}}{L^{2}}[4 \epsilon+\ldots] \\
& \approx 4 G \frac{m^{2} \epsilon}{L^{2}}
\end{aligned}
$$

If we rewrite this as an acceleration and substitute back the definition of $\epsilon$, then we have

$\ddot{x}=\frac{4 G m}{L^{3}} x$.

The solution to this equation is the exponential:

$x=\mathrm{e}^{\sqrt{\frac{4 G m}{L^{3}}} t}$.

This is qualitatively what is expected from a cosmological constant: an exponentially growing length scale.

Could this have any relation to exponential growth in the scale factor $a(t)$, i.e. could it provide a cosmological constant, or at least a form of dark energy consistent with that observed?

Before discussing this question in the next section, we first note that, given the causal contact assumption (4), we could expect that not only the closest topological image would have an effect on a cluster, but also successive images. 
Equation (2) for the first $N$ successive pairs of topological images then becomes:

$$
\begin{aligned}
F \approx & G m^{2}\left[\frac{1}{(L-x)^{2}}-\frac{1}{(L+x)^{2}}\right] \\
& +\frac{1}{(2 L-x)^{2}}-\frac{1}{(2 L+x)^{2}}+\ldots \\
\approx & \frac{4 G m^{2}}{L^{2}} \epsilon \sum_{i=1}^{N} \frac{1}{i^{3}} \\
\approx & 4.8 G \frac{m^{2} \epsilon}{L^{2}}
\end{aligned}
$$

for $N \gg 1$.

This is only a small correction to Eq. (4) - because an effect weakening with the cube of the distance decreases rapidly.

\subsubsection{Effect relative to the cosmic web}

As mentioned above, in Sect. 2.2, the geometry for an object slowly starting to fall towards a massive cluster, i.e. falling towards a dense node of the cosmic web of density perturbations, is that shown in Fig. 3.

The equation for the long-distance component of acceleration is algebraically the same as for cluster self-gravity, i.e. as in Eq. (2), except that there is also an acceleration term $-G \frac{m}{x^{2}}$ caused by the local copy of the cluster near the test object, since we are interested in long-distance effects, and for convenience, we divide by the mass of the test object:

$$
\begin{aligned}
\ddot{x} & \approx-G \frac{m}{x^{2}}+G m\left[\frac{1}{(L-x)^{2}}-\frac{1}{(L+x)^{2}}\right] \\
& \approx-G \frac{m}{x^{2}}+\frac{4 G m}{L^{3}} x .
\end{aligned}
$$

The first term in the second line of Eq. (8) represents local attraction, i.e. Newtonian gravity as it is normally thought of, inversely proportional to distance, but the second term is a long-distance term, identical to Eq. (5), directly proportional to distance.

However, although this long-distance term is algebraically identical to the right-hand side of Eq. (5), the interpretation is different.

Instead of the equation representing a high mass cluster which has been perturbed from its position and is exponentially accelerated away from its initial position, in this case we have a test particle which feels (in addition to local acceleration towards the cluster potential well) an acceleration away from its nearby (but multiply imaged) cluster potential well.

Again, this is qualitatively similar to the effect of a cosmological constant, since it is repulsive. Moreover, it is an effect additional to local gravitational terms - which is what would be required of something providing a cosmological constant.

However, since we make assumptions about the cosmological epoch, the effect is unlikely to be constant with time, so it is more accurate to say that the effect is qualitatively similar to the effect of a positive dark energy term.

Note that, in this case, if we consider only the period of infall, before any path crossing or virialisation occurs, i.e. when $x$ decreases with time, then force $F$ slows down the rate at which the test particle falls towards the cluster. Nevertheless, the particle is starting to fall towards the cluster - in comoving coordinates so, if an individual test particle has started falling towards the cluster in physical coordinates, then the effect from the topological images will exponentially decrease with time, as the particle approaches the cluster. This is not a problem, since we are not interested in following the path of any individual particle over time, and since we are most interested in a phenomenon which can be related to the system of comoving coordinates itself, i.e. to the acceleration equation of the FLRW model.

\subsection{Three-dimensional analysis}

We generalise the above calculation to an arbitrary displacement of the test particle from the cluster, in a direction not necessarily aligned with the generator $g_{x}$, and including Newtonian approximation gravitational attraction from multiple images generated by orthogonal generators $g_{y}$ and $g_{z}$, for the case of the three-torus $\mathbb{T}^{3}$. We generalise the length $L$ of $g_{x}$ by writing lengths $L_{\mathrm{a}} \equiv L$, $L_{\mathrm{e}}, L_{\mathrm{u}}$ (Lehoucq et al. 1996) for the lengths of $g_{x}, g_{y}$ and $g_{z}$ respectively. Define the displacement vector

$\boldsymbol{r}:=(x, y, z)$

of modulus $r$.

We continue to assume $x, y, z \ll L_{\mathrm{a}}, L_{\mathrm{e}}, L_{\mathrm{u}}$ in order to make first order approximations from Taylor expansions.

The generalisation of Eq. (8) is then

$$
\begin{aligned}
& \ddot{\boldsymbol{r}}=-G m \frac{\boldsymbol{r}}{r^{3}}+\ddot{\boldsymbol{r}}_{\text {topo }} \\
&=-G m \frac{\boldsymbol{r}}{r^{3}}+ \\
& G m \sum_{(i, j, k) \neq(0,0,0)} \frac{\left(i L_{\mathrm{a}}-x, j L_{\mathrm{e}}-y, k L_{\mathrm{u}}-z\right)}{\left[\left(i L_{\mathrm{a}}-x\right)^{2}+\left(j L_{\mathrm{e}}-y\right)^{2}+\left(k L_{\mathrm{u}}-z\right)^{2}\right]^{3 / 2}}
\end{aligned}
$$

where we write $\ddot{\boldsymbol{r}}_{\text {topo }}$ for the residual acceleration due to the topological images of the cluster.

Since the forces add vectorially by orthogonal components, we can first calculate the sum for the same two images we used before, i.e. for the two images of the cluster along the $x$-axis, at $i= \pm 1, j=k=0$. This is illustrated in Fig. 4 .

\subsection{1. $x$ component for $i= \pm 1, j=k=0$}

The $x$ component of the two terms $i= \pm 1, j=k=0$ from Eq. (10) is, using $\epsilon:=x / L_{\mathrm{a}}$ as above,

$$
\begin{aligned}
\frac{1}{G m} & \left(\left(\ddot{\boldsymbol{r}}_{\text {topo }}\right)_{i= \pm 1, j=k=0}\right)_{x} \\
= & \frac{L_{\mathrm{a}}-x}{\left[\left(L_{\mathrm{a}}-x\right)^{2}+y^{2}+z^{2}\right]^{3 / 2}}+\frac{-L_{\mathrm{a}}-x}{\left[\left(-L_{\mathrm{a}}-x\right)^{2}+y^{2}+z^{2}\right]^{3 / 2}} \\
= & \frac{L_{\mathrm{a}}^{-2}(1-\epsilon)}{\left[(1-\epsilon)^{2}+\left(\frac{y}{L_{\mathrm{a}}}\right)^{2}+\left(\frac{z}{L_{\mathrm{a}}}\right)^{2}\right]^{3 / 2}} \\
& +\frac{L_{\mathrm{a}}^{-2}(-1-\epsilon)}{\left[(-1-\epsilon)^{2}+\left(\frac{y}{L_{\mathrm{a}}}\right)^{2}+\left(\frac{z}{L_{\mathrm{a}}}\right)^{2}\right]^{3 / 2}} \\
= & L_{\mathrm{a}}^{-2}\left[(1-\epsilon)(1-2 \epsilon+\ldots)^{-3 / 2}\right. \\
& \left.+(-1-\epsilon)(1+2 \epsilon+\ldots)^{-3 / 2}\right] \\
= & L_{\mathrm{a}}^{-2}[(1-\epsilon)(1+3 \epsilon+\ldots)+(-1-\epsilon)(1-3 \epsilon+\ldots)] \\
= & L_{\mathrm{a}}^{-2}[(1+2 \epsilon+\ldots)+(-1+2 \epsilon+\ldots)] \\
= & 4 \epsilon L_{\mathrm{a}}^{-2} \\
= & 4 x L_{\mathrm{a}}^{-3} .
\end{aligned}
$$




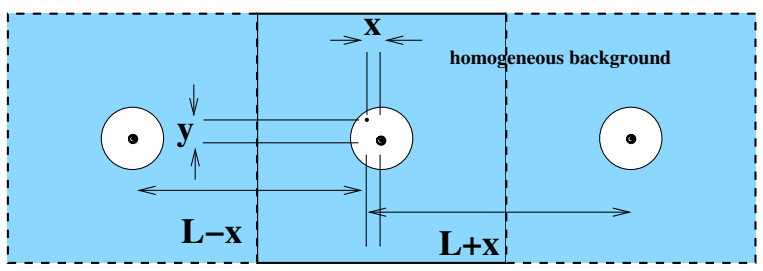

Fig. 4. Effect relative to the cosmic web: three-dimensional version of Fig. 3. The small test object is separated from the cluster by $x$ along the direction of the generator $g_{x}$, and by $y$ and $z$ in orthogonal directions. The $z$ direction is not shown. Topological images of the cluster at $i=$ $\pm 1, j=k=0$ are shown.

Unsurprisingly, to first order, this is the same result as in Eq. (8). The $y$ and $z$ displacements do not affect the residual force in the $x$ direction, they only have second order effects.

\subsection{2. $y, z$ components for $i= \pm 1, j=k=0$}

The $y$ and $z$ components of the $i= \pm 1, j=k=0$ terms are calculated as follows.

$$
\begin{aligned}
\frac{1}{G m} & \left(\left(\ddot{\boldsymbol{r}}_{\text {topo }}\right)_{i= \pm 1, j=k=0}\right)_{y} \\
= & \frac{-y}{\left[\left(L_{\mathrm{a}}-x\right)^{2}+y^{2}+z^{2}\right]^{3 / 2}}+\frac{-y}{\left[\left(-L_{\mathrm{a}}-x\right)^{2}+y^{2}+z^{2}\right]^{3 / 2}} \\
= & -y L_{\mathrm{a}}^{-3}\left\{\left[(1-\epsilon)^{2}+\left(\frac{y}{L_{\mathrm{a}}}\right)^{2}+\left(\frac{z}{L_{\mathrm{a}}}\right)^{2}\right]^{-3 / 2}\right. \\
& \left.+\left[(-1-\epsilon)^{2}+\left(\frac{y}{L_{\mathrm{a}}}\right)^{2}+\left(\frac{z}{L_{\mathrm{a}}}\right)^{2}\right]^{-3 / 2}\right\} \\
= & -y L_{\mathrm{a}}^{-3}\left[(1-2 \epsilon+\ldots)^{-3 / 2}+(1+2 \epsilon+\ldots)^{-3 / 2}\right] \\
= & -y L_{\mathrm{a}}^{-3}[(1+3 \epsilon+\ldots)+(1-3 \epsilon+\ldots)] \\
\approx & -2 y L_{\mathrm{a}}^{-3},
\end{aligned}
$$

and similarly,

$$
\frac{1}{G m}\left(\left(\ddot{\boldsymbol{r}}_{\text {topo }}\right)_{i= \pm 1, j=k=0}\right)_{z} \approx-2 z L_{\mathrm{a}}^{-3}
$$

Unsurprisingly, this yields a weak net force pulling the test object back towards the $x$-axis joining the two topological images of the cluster.

However, what may be surprising is that this force increases in amplitude as the test object's $y$ (or $z$ ) separation increases. For the $x$-component, it is obvious that as the test object becomes less and less symmetrically placed between the two topological images, i.e. as $x$ increases, the residual force towards the closer image should increase in amplitude. But how is it possible that as the test object moves further from the $x$-axis, i.e. as $y$ increases, the residual force pulling it back to the plane increases?

The explanation is in the vectorial nature of the addition of forces. (A Euclidean covering space is presumed throughout.) The force in the $-y$ direction is only a component of a total, vectorial force. For very small $y$, the two force vectors towards the two topological images almost completely cancel since they are nearly perfectly parallel. As $y$ increases, these two vectors become less parallel and cancel less completely, so although their individual (scalar) amplitudes decrease, the $y$ component of their vector sum increases.

\subsubsection{Vectorial residual force for $x$ axis and for all images}

Equations (11)-(13) yield the total vectorial residual acceleration for the $i= \pm 1, j=k=0$ images of the cluster, i.e. for the closest $x$-axis images:

$\left(\ddot{\boldsymbol{r}}_{\text {topo }}\right)_{i= \pm 1, j=k=0}=G m \frac{(4 x,-2 y,-2 z)}{L_{\mathrm{a}}^{3}}$.

By symmetry, the resultant residual acceleration for the closest images from all three axes is:

$$
\begin{gathered}
\left(\ddot{\boldsymbol{r}}_{\text {topo }}\right)_{(i, j, k) \in\{( \pm 1,0,0),(0, \pm 1,0),(0,0, \pm 1)\}} \\
=G m\left[\frac{(4 x,-2 y,-2 z)}{L_{\mathrm{a}}^{3}}+\frac{(-2 x, 4 y,-2 z)}{L_{\mathrm{e}}^{3}}+\frac{(-2 x,-2 y, 4 z)}{L_{\mathrm{u}}^{3}}\right] \\
=2 G m\left[x\left(\frac{2}{L_{\mathrm{a}}^{3}}-\frac{1}{L_{\mathrm{e}}^{3}}-\frac{1}{L_{\mathrm{u}}^{3}}\right), y\left(\frac{2}{L_{\mathrm{e}}^{3}}-\frac{1}{L_{\mathrm{a}}^{3}}-\frac{1}{L_{\mathrm{u}}^{3}}\right),\right. \\
\left.z\left(\frac{2}{L_{\mathrm{u}}^{3}}-\frac{1}{L_{\mathrm{a}}^{3}}-\frac{1}{L_{\mathrm{e}}^{3}}\right)\right] .
\end{gathered}
$$

If $L_{\mathrm{a}}=L_{\mathrm{e}}=L_{\mathrm{u}}$, these terms cancel and the resultant residual acceleration is zero.

By symmetry, each successively distant orthogonal, equidistant 8-tuplet of topological images in Eq. (10) also contributes a zero sum if $L_{\mathrm{a}}=L_{\mathrm{e}}=L_{\mathrm{u}}$. Numerical calculation of the contributions of other symmetrical $n$-tuplets of topological images distant from the $(0,0,0)$ image indicates that these also contribute zero to the sum, so that the full (first order) sum is zero.

This shows a three-dimensional effect different to that from the $x$-axis calculation alone: the residual gravitational acceleration induced by multiple images disappears if space is an "isotropic $\mathbb{T}^{3}$ " model, in the sense that the three lengths of the fundamental domain are equal.

On other hand, in a "slightly anisotropic $\mathbb{T}^{3}$ " model, the residual gravity due to multiple images does not totally disappear. In order to consider the case in which the three side lengths of the fundamental domain are slightly unequal, define

$\delta_{\mathrm{e}}:=\frac{L_{\mathrm{e}}}{L_{\mathrm{a}}}-1, \quad \delta_{\mathrm{u}}:=\frac{L_{\mathrm{u}}}{L_{\mathrm{a}}}-1$.

Then we have

$$
\begin{aligned}
\frac{2}{L_{\mathrm{a}}^{3}}-\frac{1}{L_{\mathrm{e}}^{3}}-\frac{1}{L_{\mathrm{u}}^{3}} & =L_{\mathrm{a}}^{-3}\left[2-\left(1+\delta_{\mathrm{e}}\right)^{-3}-\left(1+\delta_{\mathrm{u}}\right)^{-3}\right] \\
& =L_{\mathrm{a}}^{-3}\left[2-\left(1-3 \delta_{\mathrm{e}}+\ldots\right)-\left(1-3 \delta_{\mathrm{u}}+\ldots\right)\right] \\
& =3 L_{\mathrm{a}}^{-3}\left(\delta_{\mathrm{e}}+\delta_{\mathrm{u}}\right)
\end{aligned}
$$

and similarly

$\frac{2}{L_{\mathrm{e}}^{3}}-\frac{1}{L_{\mathrm{a}}^{3}}-\frac{1}{L_{\mathrm{u}}^{3}}=3 L_{\mathrm{a}}^{-3}\left(-2 \delta_{\mathrm{e}}+\delta_{\mathrm{u}}\right)$,

so that

$$
\begin{aligned}
& \left(\ddot{\boldsymbol{r}}_{\text {topo }}\right)_{(i, j, k) \in\{( \pm 1,0,0),(0, \pm 1,0),(0,0, \pm 1)\}} \\
& \quad=6 G m L_{\mathrm{a}}^{-3}\left[x\left(\delta_{\mathrm{e}}+\delta_{\mathrm{u}}\right), y\left(-2 \delta_{\mathrm{e}}+\delta_{\mathrm{u}}\right), z\left(\delta_{\mathrm{e}}-2 \delta_{\mathrm{u}}\right)\right] .
\end{aligned}
$$

For the case $L_{\mathrm{a}}=L_{\mathrm{e}}\left(\right.$ i.e. $\left.\delta_{\mathrm{e}}=0\right)$, this becomes

$\left(\ddot{\boldsymbol{r}}_{\text {topo }}\right)_{(i, j, k) \in\{( \pm 1,0,0),(0, \pm 1,0),(0,0, \pm 1)\}}=6 G m \delta_{\mathrm{u}} L_{\mathrm{a}}^{-3}(x, y,-2 z)$

while for the case $L_{\mathrm{e}}=L_{\mathrm{u}}$ (i.e. $\delta_{\mathrm{e}}=\delta_{\mathrm{u}}$ ), this becomes

$\left(\ddot{\boldsymbol{r}}_{\text {topo }}\right)_{(i, j, k) \in\{( \pm 1,0,0),(0, \pm 1,0),(0,0, \pm 1)\}}=6 G m \delta_{\mathrm{u}} L_{\mathrm{a}}^{-3}(2 x,-y,-z)$. 
This differs from the acceleration in Eq. (14) by a factor of $3 \delta_{\mathrm{u}}$. In other words, if the long fundamental dimensions are equal to one another and one-third greater than the short dimension, then the linearised (in $\delta_{\mathrm{u}}$ ) estimate of the effect summed from the three directions, in Eq. (21), is as large as if the two larger dimensions were infinite, as is effectively represented by Eq. (14). Clearly, this implies that the approximation is valid only for $\delta_{\mathrm{u}} \ll 1 / 3$.

In each case, this qualitative behaviour is similar to a positive dark energy term in the direction of the shorter fundamental length(s), and a negative dark energy term in the direction of the longer fundamental length(s), indicating that the effect would tend to equalise the three fundamental lengths of a $\mathbb{T}^{3}$ model.

Numerical evaluation of further terms in the sum on the right-hand side of Eq. (10) indicates that these modify the total sum only slightly.

\section{Discussion}

Is there any relation between the effect found here and dark energy? Would this effect really tend to equalise the three fundamental lengths of a $\mathbb{T}^{3}$ model?

For simplicity, consider the $x$-axis case and the cluster or test object displaced along the $x$-axis, for the $\mathbb{T}^{1}$ model.

What has been shown so far is that, under the assumptions listed above, the gravitational effect due to multiple topological imaging provides an acceleration proportional to displacement, i.e. constant $\ddot{x} / x$, where either (Sect. 2.1) this is the total acceleration for a given massive object towards its closer topological image, or, (Sect. 2.2) it is the total long-distance induced acceleration (in addition to locally induced acceleration) for a test object "falling" towards a given massive object.

The calculation itself is made in comoving space: the result of Sect. 2.1, for self-gravity of a cluster towards itself, is that a perturbed, large massive object is slightly accelerated in the direction of its closest image, and this acceleration is proportional to the displacement from the initial position in comoving space, so that the displacement increases exponentially. Since we are working within the comoving frame, it is not obvious, in the Newtonian approximation, how to relate this to a modification in the equations for the growth of the scale factor itself with cosmological time.

On the other hand, since the cosmic web is, on average, fixed in the comoving frame, it may be possible to interpret the second case (Sect. 2.2), of test objects "falling" towards the most massive objects in the cosmic web, in terms of a dark energy term.

For objects still distant from and falling into massive clusters, could the additional force term of Eq. (5), as shown in Fig. 3, provide the pressure term in the FLRW acceleration equation,

$\frac{\ddot{a}}{a}=-\frac{4 \pi G}{3} \rho\left(1+3 \frac{p}{\rho c^{2}}\right)$,

where $a$ is the scale factor, in order to mimic dark energy?

\subsection{Amplitude of cosmo-topological gravity relative to the cosmic web}

An heuristic Newtonian derivation of the acceleration equation in this case, for simplicity, treating the $x$-axis case, for the $\mathbb{T}^{1}$ model, follows from Eq. (8), where we define $\chi$ to be a fixed length in the comoving reference frame so that $x=a \chi$. $L$ has already been defined to be a comoving length, but in the above equations we implicitly used $a=1$; here, since we want expressions valid at arbitrary values of the scale factor, not only $a=1$, we write $a L$ rather than $L$.

$$
\begin{aligned}
\ddot{a} \chi & =\ddot{x} \\
& \approx-G \frac{m}{x^{2}}+G \frac{4 m}{(a L)^{3}} x \\
& =-G \frac{4 \pi \rho}{3} a \chi+G \frac{4 \pi \rho}{3} \frac{4 a^{3} \chi^{3}}{a^{3} L^{3}} a \chi
\end{aligned}
$$

if we estimate that the cluster mass was obtained from matter spread at the mean density $\bar{\rho}$ throughout a sphere of radius $x$.

Note that assumptions (5) and (6) are crucial here, since we assume that the relevant matter density, both locally and at long distance, is that contained inside of local and topologically imaged, distant spheres, fixed within comoving coordinates, around the cluster centre.

Dividing both sides of Eq. (23) by $x=a \chi$ yields

$\frac{\ddot{a}}{a}=-G \frac{4 \pi \rho}{3}\left(1-\frac{4 \chi^{3}}{L^{3}}\right)$.

Using the standard notation for a dark energy component, $w:=$ $p /\left(\rho c^{2}\right)$, we can rewrite this

$w=-\frac{4}{3}\left(\frac{\chi}{L}\right)^{3} \sim-\left(\frac{\chi}{L}\right)^{3}$

Since we are interested in objects "falling" (in comoving coordinates) towards the nodes of the cosmic web, i.e. at most a few tens of Mpc from those nodes, then for a universe side length as large as the diameter of the surface of last scattering, $L=20 h^{-1} \mathrm{Gpc}$, we have

$w \sim-10^{-9}$.

So, for the $x$-axis case in a $\mathbb{T}^{1}$ model, while cosmo-topological gravity has the right algebraic characteristics in this heuristic approach, with the assumptions listed above, to provide an acceleration similar to a dark energy term, its amplitude in the present-day Universe is certainly too small to be significant, except possibly for extremely high-resolution $N$-body simulations of the formation of structure in the Universe.

Of course, if the length scale of large scale structure were nearly as large as that of the Universe itself, i.e. if $\chi \sim L$, then the amplitude of this effect would be much larger. However, this would not be physically realistic according to our understanding of structure formation.

As shown in Sect. 3.2, if we consider a displacement of the test particle in an arbitrary direction relative to the generator vector, and if we sum the contributions from the three directions in a $\mathbb{T}^{3}$ model of nearly equal fundamental lengths in the three directions, then a similar heuristic argument, using an anistropic scale factor

$\boldsymbol{a}(t)=\left(a_{\mathrm{a}}, a_{\mathrm{e}}, a_{\mathrm{u}}\right)(t)$

implies a similar, but anisotropic, effective acceleration term approximately $\delta$ times weaker than for a $\mathbb{T}^{1}$ model (Eqs. (8), (10), (21)), where $\delta$ is the fractional difference in fundamental lengths.

In contrast to the $\mathbb{T}^{1}$ case, if the three lengths are exactly equal, then this effect cancels to zero. The fundamental lengths must be slightly unequal in order for there to be an effect. 
Interestingly, as noted in Sect. 3.2.3, this effect will be anisotropic in such a way as to oppose the anisotropy of the three fundamental lengths, tending to push the three fundamental lengths towards equality. This is probably the first time that such an effect tending to induce $L_{\mathrm{a}}=L_{\mathrm{e}}=L_{\mathrm{u}}$ for a $\mathbb{T}^{3}$ model has been found. The latter is often assumed for simplicity and aesthetic reasons, but here we seem to have a physical motivation for this equality as a stable equilibrium rather than as an arbitrary assumption.

\subsection{Caveat: Time-varying mass of cluster}

A minor caveat to note for cosmo-topological gravity is that the mass $m$ is not constant with time in our model: gravitational collapse will continue and successively larger and larger objects will form. However, it has already been noted above that this effect is linked to the cosmological epoch, and is thus likely to vary with time, so this is not necessarily a strong argument against some role for this effect as a dark energy term: the low amplitude of the effect is a much greater problem.

\subsection{Caveat: $\chi$ not constant within large scale structure unit}

Another minor caveat, in addition to the anisotropy of the effect, is that this effect will vary with distance $\chi$ from the nearest big cluster (node of the cosmic web).

This implies that the spatially averaged value of $w \sim-\left(\frac{x}{L}\right)^{3}$ would be needed before comparing a theoretical $w$ value with an observed value, such as the presently estimated value of $w \approx-1$.

Since most cosmological observations relevant to estimating the parameters of the metric are in practice averaged out over scales larger than that of large scale structure, i.e. on scales $\gg 100 h^{-1} \mathrm{Mpc}$, this is not a problem.

However, it is interesting to see how the ratio of the topological acceleration term to the local acceleration term towards the dominant nearby cluster varies as a function of the length scale towards nearby clusters. This has been presented below in Sect. 4.4.

\subsection{Numerical test}

As a numerical check on analytical calculations of this effect, the zero redshift output data file for a "Lambda CDM" model of the "Hubble Volume" one billion particle simulation of the present-day distribution of galaxy clusters made by the Virgo Supercomputing Consortium (Evrard et al. 2002) is used here.

In other words, a present-day synthetic distribution of clusters for a model universe with $\Omega_{\mathrm{m}}=0.3, \Omega_{\Lambda}=0.7, H_{0}=$ $70 \mathrm{~km} \mathrm{~s}^{-1} \mathrm{Mpc}^{-1}$ and a CDM (cold dark matter) initial power spectrum of density perturbations consistent with these parameters, is used ${ }^{1}$. The data file contains mass and positions of "clusters" detected as virialised groups of particles by using a friendsof-friends (FOF) algorithm with a linking length of $b=0.164$. Individual particles have masses of $2.24 \times 10^{12} h^{-1} M_{\odot}$.

The side length of the cube is $3 h^{-1} \mathrm{Gpc}$. This length scale is about six times smaller than the likely minimum value of $L$,

\footnotetext{
1 See: http://www .mpa-garching.mpg.de/Virgo/hubble.html; the file is downloadable at the time of submission as http://ln-s.net/E3s or as http://www.mpa-garching.mpg.de/Virgo_Data/hubble_ cluster/snapshot/lcdm/cluster_fof_lcdm_zQ_bo.164.tar.gz.
}

but is about one and a half orders of magnitude larger than the length scale of "large scale structure", i.e. the scales on which clusters form voids and walls, so it should be good enough to qualitatively test the result of analytical calculations.

For each test point, the vector accelerations as described in Eq. (10) are calculated. That is, the standard gravitational acceleration $-G m \frac{r}{r^{3}}$ towards the cluster whose gravitational pull is strongest at the test point and the "topological" gravitational component $\ddot{\boldsymbol{r}}_{\text {topo }}$ for the topological images of this cluster are calculated. This is done:

(i) for the $\mathbb{T}^{1}$ case (2 adjacent topological images),

(ii) for the $\mathbb{T}^{3}$ case ( 8 adjacent topological images) with three exactly equal fundamental lengths, and

(iii) for the $\mathbb{T}^{3}$ case with $y$ and $z$ axes' fundamental lengths a small fraction $\delta_{\mathrm{e}}=\delta_{\mathrm{u}}=0.1$ larger than the $x$ axis fundamental length.

From Eqs. (14) and (21) and the derivation leading to Eq. (25), we would expect these three ratios (for the $x$ axis direction which dominates, without loss of generality) to be approximately:

(i) $4\left(\frac{\chi}{L}\right)^{3}$,

(ii) $\ll 4\left(\frac{\chi}{L}\right)^{3}$, and

(iii) $12 \delta_{\mathrm{e}}\left(\frac{\chi}{L}\right)^{3}$

respectively.

Figures 5-8 show that in a realistic simulation on a scale just one order of magnitude smaller than the diameter of the last scattering surface (Evrard et al. 2002), and on scales far enough from individual clusters to be participating in the linear regime of density perturbation theory (i.e. still expanding with the Hubble flow), the ratio of the "topological" acceleration to the normal local acceleration is consistent with these three expressions.

Of course, since the size of the simulation is $3 h^{-1} \mathrm{Gpc}$ rather than a more realistic scale of $20 h^{-1} \mathrm{Gpc}$, the acceleration ratios in these figures should be reduced by approximately $(20 / 3)^{3}$, i.e. by a factor of about 300 , in agreement with the estimate presented below in Eq. (26).

\subsection{Assumptions (5), (6), the topological one-body and two-body problems}

Another caveat, probably more important, is that the calculation we have made, based on assumptions (5) and (6) (Sect. 2.1), is equivalent to what we might call the "topological one-body and two-body problems".

Naiively, it may seem that both the Newtonian and relativistic versions of gravity exclude gravitational self-interaction of a single, massive, point-sized body: a gravitational interaction between $N$ bodies normally requires $N \geq 2$ bodies.

However, this intuition relies on the implicit assumption that space is an infinite, simply connected Euclidean space.

In a multiply connected space, a single body can be thought of - in the covering space - as a set of multiple bodies. Although $N=1$ bodys exists in a true physical sense and in the fundamental domain, $N \gg 1$ bodies exist in the covering space, which is the simplest space in which to calculate gravitational interactions.

This is why it is possible for a single body to have gravitational self-interactions according to the standard Newtonian approximation of gravity. Hence, the "one-body problem" is a serious dynamical problem, despite its apparent absurdity. 


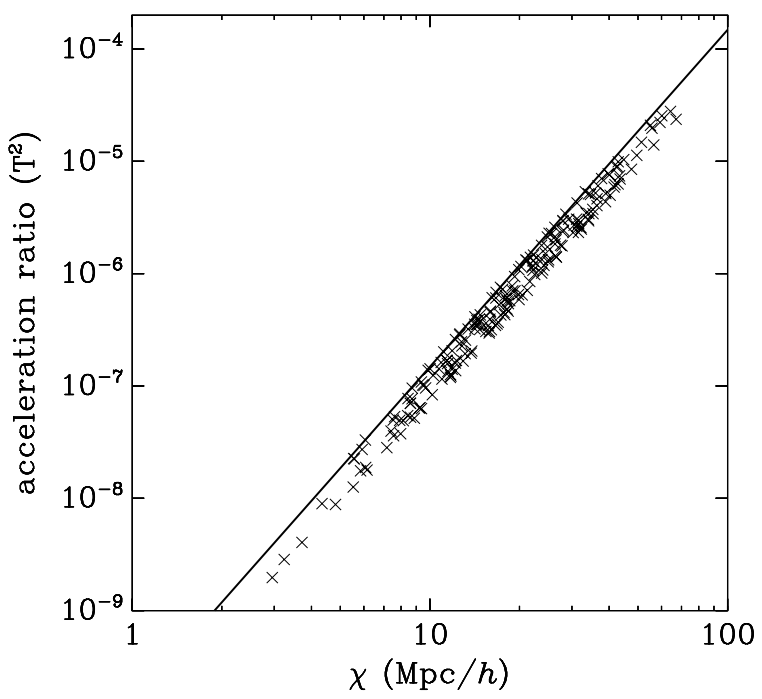

Fig. 5. Ratio of the topological acceleration effect (the effect relative to the cosmic web) over the normal gravitational infall, shown as a function of the distance of a test point to the cluster exerting the greatest gravitational pull on it, using a Hubble Volume simulation by the Virgo Supercomputing Consortium (see Sect. 4.4). This plot shows the $\mathbb{T}^{1}$ case showing the ratios of the modulus of the accelerations. The line shows the analytical estimate $4\left(\frac{\chi}{L}\right)^{3}$ (see Eqs. (14) and (25)).

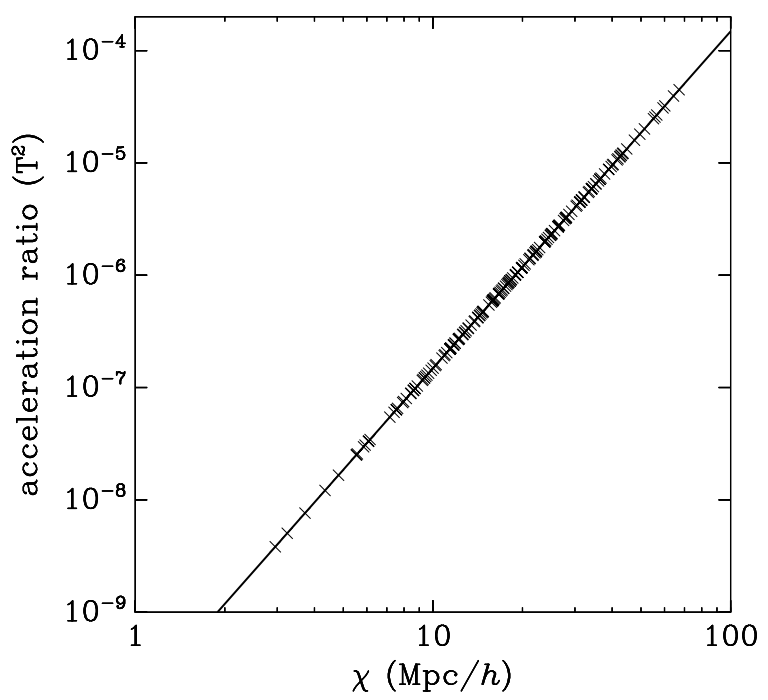

Fig. 6. As per Fig. 5, showing the (negative of the) topological-tonormal ratio of the $x$ axis accelerations in the $\mathbb{T}^{1}$ case and the function $4\left(\frac{x}{L}\right)^{3}$. All test points were found (as expected) to have opposite signs in the $x$ direction topological and normal accelerations; hence, the negative was used.

The method of calculating these interactions depends on how realistic our universe model is.

The assumptions (5) and (6) permit a relatively simple calculation, and are equivalent to assuming a flat (even though mostly empty) space which contains just one (or two) objects, i.e. our assumptions are equivalent to a very simple, though physically unrealistic, model.

Also, the distant, supra-SLS images of a cluster are notyet collapsed perturbations, so approximating them as single,

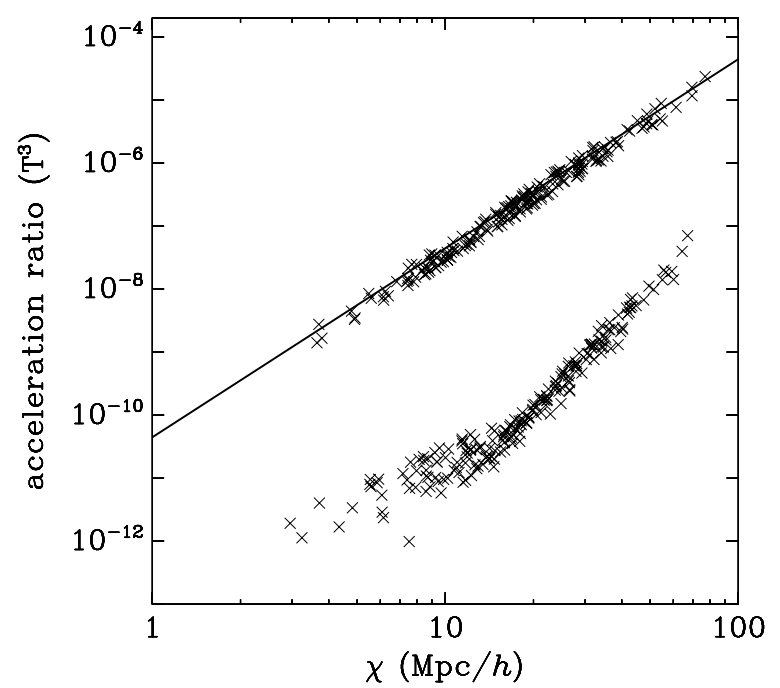

Fig. 7. As per Fig. 5, showing the ratios of the modulus of the accelerations for the $\mathbb{T}^{3}$ case. The lower scattering of points show the case with three equal fundamental lengths and the upper scattering of points shows the case with $\delta_{\mathrm{e}}=\delta_{\mathrm{u}}=0.1$. The line shows the analytical estimate $12 \delta_{\mathrm{e}}\left(\frac{\chi}{L}\right)^{3}$ (see Eqs. (21) and (25)).

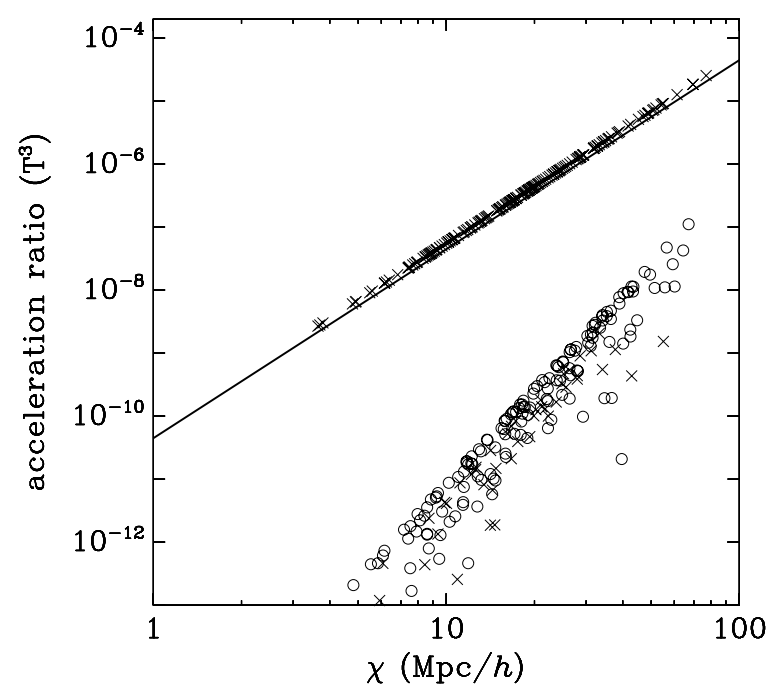

Fig. 8. As per Fig. 7, showing the (negative of the) ratios of the $x$ axis accelerations in the $\mathbb{T}^{3}$ case with equal fundamental lengths (lower " $\times$ " points) and unequal lengths (upper " $X$ " points). Positives of the ratios, i.e. when a test point is attracted by the topological residual force in the same direction as the local gravitational force, are shown as circles. This latter situation only occurs for the case of equal fundamental lengths, where the topological force cancels perfectly according to the analytical calculation. The line shows $12 \delta_{\mathrm{e}}\left(\frac{\chi}{L}\right)^{3}$.

point-like objects, while ignoring matter outside the radius from which they form is somewhat arbitrary.

Is it possible to make a more precise calculation which avoids assumptions (5) and (6)? Numerical calculations would be possible in principle, and would provide a good followup to our present result.

However, these are unlikely to be easy, and would be different from standard $N$-body simulations because of assumptions (7) and (8). Standard $N$-body simulations use various numerical approximation techniques in order to make the 
calculation time short enough to be practical, and, de facto make at least one of the following two asssumptions:

(i) instead of assumption (4), assume that something like inflation has not occurred

(ii) instead of assumptions (5) and (6), assume that all contributions to the local gravitational potential due to long-range, supra-SLS distance scale density perturbations perfectly cancel out.

Since inflation scenarios are good candidates for providing some ingredient of the correct model of the Universe, there is a good chance that assumption (i) is incorrect.

Moreover, it is not clear to what extent assumption (ii) is a good approximation.

Newtonian gravititational attraction between two point objects decreases according to the inverse square of the distance, i.e. as $\propto r^{-2}$, but the mass in successive spherical shells of equal thickness increases as $r^{2}$ : an anisotropy of fixed (small) solid angular size $\cos \theta \mathrm{d} \phi \mathrm{d} \theta$ at all radii would provide equal attractive forces from each successive shell, no matter how distant.

This is no problem in a perfectly homogeneous universe: hence, the FLRW solution of the Einstein field equations.

But in a universe with perturbations, i.e. a "slightly" inhomogeneous, anisotropic universe, the contributions do not perfectly cancel.

In a nearly FLRW universe, i.e. one containing a spectrum of density perturbations, the degree to which the long-distance terms cancel most likely depends on the full nature of the perturbation spectrum.

Farrar \& Melott (1990) suggest from their $N$-body calculations that assumption (ii) is correct in the case of a multiply connected flat space (of equal fundamental lengths). Further work in this direction would be interesting, in particular by introducing the physically standard assumption (not used explicitly in normal $N$-body simulations), i.e. our assumption (7), that $c_{\mathrm{GW}}$ is finite and of the standard value $c_{\mathrm{GW}}=c_{\mathrm{ST}}=c_{\mathrm{GR}}=c_{\mathrm{EM}}$. Testing the effects of slightly unequal fundamental lengths would also be interesting.

\subsubsection{Inhomogeneities and the Einstein-Hilbert equations}

As has been pointed out for many years, e.g. Sect. 7, Ellis \& van Elst (1999) and references therein, finding an exact inhomogeneous solution to the Einstein-Hilbert equations, and then averaging this to find a "mean density" homogeneous solution, is not mathematically equivalent to adding perturbations to the exact FLRW (homogeneous) solution to the Einstein-Hilbert equations. The latter, approximate approach gives a good match to observations, but this does not prove that the difference between the exact and perturbative approaches is negligible.

Buchert \& Carfora $(2002,2003)$ have been studying this problem in depth for several years and have noted that the difference between the perturbative approach and the exact (but still developing) approach leads to a "backreaction" term (and also a curvature term) which may have an effect on scales ranging from that of a massive galaxy cluster possibly up to superHubble length scales. Recent discussions (Kolb et al. 2005,b) indicate that this presently seems, pending full calculation, to be a viable candidate for a dark energy term, without the need for, e.g. adding any scalar fields, based on the backreaction term from $s u b$-Hubble length perturbations.

In a qualitative sense, this is similar to the effect discussed in this paper, except that we are primarily interested here in the effect of perturbations on gravity in the case of non-trivial global topology.

Buchert \& Ehlers (1997) and Ehlers \& Buchert (1997) presented work closely related to what is presented here, considering both $\mathbb{T}^{3}$ and $\mathbb{R}^{3}$ models, for the Newtonian case of perfectly isotropic expansion:

$a_{\mathrm{a}}(t)=a_{\mathrm{e}}(t)=a_{\mathrm{u}}(t) \quad \forall t$.

For the $\mathbb{T}^{3}$ case, they found a consistent result with our first order result, i.e. that for a compact space admitting a global Hubble flow, i.e. if the average shear and rotation are zero (Appendix A, Buchert \& Ehlers 1997; see also four equivalent statements related to this in Sect. 3.4 of Buchert \& Ehlers 1997), then the perturbations have no effect on the rate of expansion according in the Newtonian approximation.

Given that our heuristic calculation suggests that for a slight anisotropy in the three lengths scales of $\mathbb{T}^{3}$, the expansion rate should be anisotropic, it would be interesting to develop Buchert \& Ehlers (1997)'s approach further using (slightly) anisotropic scale factors, i.e. using Eq. (27) to replace Buchert \& Ehlers (1997)'s equation $V(t)=: a_{\mathcal{D}}^{3}(t)$ (just after Eq. (3)) by

$V(t)=a_{\mathrm{a}} a_{\mathrm{e}} a_{\mathrm{u}}(t)$

and following through, using the definition in Eq. (16). The related work in Ehlers \& Buchert (1997) could be reworked starting from their Eq. (15).

Just as perfect homogeneity is certainly wrong, perfect isotropy in the expansion rate is probably also just an approximation to a more accurate model, whether or not the correct model is closer to $\mathbb{T}^{3}$ or $\mathbb{R}^{3}$ or another model.

\section{Conclusion}

A residual gravitational effect, which we could possibly call "cosmo-topological gravity", occurs due to distant multiple topological images in a multiply connected universe which "remembers" the gravitational potential generated by multiple topological images, which in the covering (apparent) space are located outside of the present surface of last scattering, and were causally contacted at some earlier epoch, for example due to some prior amount of inflation. A Newtonian approximation, in which the speed of transmission of gravitational information is finite (equal to the special relativistic space-time constant $c$ ), rather than infinite as in a fully Newtonian calculation, is used here.

For a low mass test object "falling" (in comoving coordinates) towards a relatively nearby (at a comoving distance $\chi$ less than a few tens of Mpc) large, massive collapsed object at the present epoch, i.e. a massive cluster of galaxies, in a 3-torus universe of side-length $L \approx 20 \mathrm{~h}^{-1} \mathrm{Gpc}$, the two closest topological images of the cluster together yield a residual Newtonian force on the test particle which locally appears as a force repelling the test particle away from (its nearby image of) the cluster, provided that we consider the test object lying along the axis joining the two closest topological images of the cluster in one of the fundamental directions, and we only consider these two images.

This residual force provides an acceleration algebraically similar to that of dark energy, but weaker by many orders of magnitude, i.e. by a factor of approximately $\left(\frac{\chi}{L}\right)^{3} \sim 10^{-9}$ at the present epoch.

A more general, three-dimensional calculation, for a test object displaced in an arbitrary direction in a 3-torus universe, 
shows that the effect cancels out to zero if the three side lengths are exactly equal. If the side lengths are slightly unequal, by a fraction $\sim \delta$, then an anisotropic dark energy term, about $\delta\left(\frac{\chi}{L}\right)^{3} \sim 10^{-9}$ times weaker than the observed dark energy (for side length $L \approx 20 \mathrm{~h}^{-1} \mathrm{Gpc}$ ), will accelerate the expansion of the shorter length(s) and decelerate the expansion of the longer length(s), tending to equalise them.

This is probably the first known physical effect which could relate the three fundamental lengths of a universe with $\mathbb{T}^{3}$ spatial sections. The equality of the three lengths in simulations of the $\mathbb{T}^{3}$ model has often been assumed, but on purely aesthetic grounds, without any physical justification.

It is clear that the effect is not significant in the present-day Universe.

Apart from developing an anisotropic version of Buchert \& Ehlers (1997)'s approach, an interesting (and challenging) followup project would be to check whether or not the same effect occurs in spherical, multiply connected universes: could the residual gravitational force due to multiple imaging have helped push the shape of the Universe into that of the Poincaré dodecahedral space (PDS) during or not long after the quantum epoch? Did it help isotropise the Universe?

Acknowledgements. SB acknowledges support from KBN Grant 1P03D 01226. The simulations used in this paper were carried out by the Virgo Supercomputing Consortium using computers based at the Computing Centre of the Max-Planck Society in Garching and at the Edinburgh parallel Computing Centre. The data are publicly available at http://www.mpa-garching.mpg.de/NumCos. Helpful comments from an anonymous referee were greatly appreciated.

\section{References}

Ahmadi, N., \& Nouri-Zonoz, M. 2005 [arXiv:gr-qc/0510100] Aurich, R., Lustig, S., \& Steiner, F. 2005a, ClassQuantGra, 22, 3443 Aurich, R., Lustig, S., \& Steiner, F. 2005b, ClassQuantGra, 22, 2061
Bagla, J. S. 2005, Current Science, 88, 1088

Barrow, J. D., \& Levin, J. 2001, Phys. Rev. A, 63, 044104

Blanlœil, V., \& Roukema, B. F., ed. 2000, Cosmological Topology in Paris 1998 (Paris: Blanlœil \& Roukema) [arXiv: astro-ph/0010170]

Buchert, T., \& Carfora, M. 2002, ClassQuantGra, 19, 6109

Buchert, T., \& Carfora, M. 2003, Phys. Rev. Lett., 90, 031101

Buchert, T., \& Ehlers, J. 1997, A\&A, 320, 1

Ehlers, J., \& Buchert, T. 1997, General Relativity and Gravitation, 29, 733

Ellis, G. F. R., \& Uzan, J.-P. 2005, Am. J. Phys., 73, 240

Ellis, G. F. R., \& van Elst, H. 1999, in Theoretical and Observational Cosmology, ed. M. Lachièze-Rey, NATO ASIC Proc., 541, 1

Evrard, A. E., MacFarland, T. J., Couchman, H. M. P., et al. 2002, ApJ, 573, 7

Farrar, K. A., \& Melott, A. L. 1990, Computers in Physics, 4, 185

Gundermann, J. 2005 [arXiv: astro-ph/0503014]

Kolb, E. W., Matarrese, S., \& Riotto, A. 2005, [arXiv: astro-ph/0511073]

Kolb, E. W., Matarrese, S., \& Riotto, A. 2006, New J. Phys., 8, 322

Lachièze-Rey, M. 1999, in Cosmological Topology in Paris 1998, Observatoire de Paris, ed. V. Blanlœil, \& B. F. Roukema [arXiv:astro-ph/0010170]

Lachièze-Rey, M., \& Luminet, J. 1995, Phys. Rep., 254, 135

Lahav, O., \& Liddle, A. 2004, Phys. Lett. B, 592, 1

Lehoucq, R., Lachièze-Rey, M., \& Luminet, J.-P. 1996, A\&A, 313, 339

Linde, A. 2004, J. Cosmology and Astro-Particle Physics, 10, 4

Luminet, J., \& Roukema, B. F. 1999, in Theoretical and Observational Cosmology, NATO ASIC Proc., 541, 117

Luminet, J., Weeks, J. R., Riazuelo, A., Lehoucq, R., \& Uzan, J. 2003, Nature, 425,593

Luminet, J.-P. 1998, Acta Cosmologica, XXIV-1, 105 [arXiv: gr-qc/9804006]

Rebouças, M. J., \& Gomero, G. I. 2004, Braz. J. Phys., 34, 1358

Roukema, B. F. 2000, Bull. Astr. Soc. India, 28, 483

Roukema, B. F. 2002, in Marcel Grossmann IX Conference on General Relativity, ed. V. G. Gurzadyan, R. T. Jantzen, \& R. Ruffini, World Scientific, Singapore, 1937 [arXiv: astro-ph/0010189]

Roukema, B. F., Peterson, B. A., Quinn, P. J., \& Rocca-Volmerange, B. 1997, MNRAS, 292, 835

Roukema, B. F., Lew, B., Cechowska, M., Marecki, A., \& Bajtlik, S. 2004, A\&A, 423,821

Starkman, G. D. 1998, ClassQuantGra, 15, 2529

Uzan, J.-P., Lehoucq, R., \& Luminet, J.-P. 1999, in Proc. of the XIXth Texas meeting, Paris 14-18 December 1998, ed. E. Aubourg, T. Montmerle, J. Paul, \& P. Peter, article No. 04/25 [arXiv: gr-qc/0005128]

Uzan, J.-P., Luminet, J.-P., Lehoucq, R., \& Peter, P. 2002, Eur. J. Phys., 23, 277 